Article

\title{
The Existence of Solutions to Nonlinear Matrix Equations via Fixed Points of Multivalued F-Contractions
}

\author{
Nawab Hussain ${ }^{1}, * \mathbb{D}$, Ghada Ali ${ }^{1}$, Iram Iqbal ${ }^{2}$ and Bessem Samet ${ }^{3}$ \\ 1 Department of Mathematics, King Abdulaziz University, P.O. Box 80203, Jeddah 21589, Saudi Arabia; \\ gbasendwah@kau.edu.sa \\ 2 Department of Mathematics, University of Sargodha, Sargodha 40100, Pakistan; iram.iqbal.uos@gmail.com \\ 3 Department of Mathematics, College of Science, King Saud University, P.O. Box 2455, \\ Riyadh 11451, Saudi Arabia; bsamet@ksu.edu.sa \\ * Correspondence: nhusain@kau.edu.sa
}

Received: 16 November 2019; Accepted: 31 January 2020; Published: 7 February 2020

check for updates

\begin{abstract}
In this paper, we set up an adequate condition for the presence of a solution of the nonlinear matrix equation. To do so, we prove the existence of fixed points for multi-valued modified F-contractions in the context of complete metric spaces, which generalize, refine, and extend several existing results in the literature. An example is accompanies the obtained results to show that derived results are a proper generalization.
\end{abstract}

Keywords: F-contraction; fixed points; matrix equation; multi-valued mapping

\section{Introduction and Preliminaries}

It has always been an attractive problem to find an adequate method to solve matrix equations because the existence of solutions of matrix equations arises in a number of applications such as in stochastic filtering, system theory, dynamic programming, control theory, statistics, ladder networks, and many other fields. In 2003, Ran and Reurings [1] obtained a sufficient condition for the presence of positive definite solution of two classes of matrix equations

$$
V=\mathcal{Q} \pm \sum_{j=1}^{f} \mathscr{C}_{j}^{*} \mathcal{F}(V) \mathscr{C}_{j}
$$

where $\mathcal{F}$ is a order-preserving (or order-reversing) mapping on $H(s), \mathcal{Q} \in \mathcal{P}(s)$ and $\mathscr{C}_{j}$ is an $s \times s$ complex matrix. Since then, many fixed point theorems have been presented by several authors to find solutions for different classes of matrix equations (see [2,3]). In [4], Berzig proved the existence and uniqueness of solution of the matrix equations of the form

$$
V=\mathcal{Q}+\sum_{i=1}^{f} \mathscr{C}_{i}^{*} V \mathscr{C}_{i}-\sum_{i=1}^{k} \mathscr{D}_{i}^{*} V \mathscr{D}_{i}
$$

In the present paper, our goal is to find a sufficient condition to determine a solution for nonlinear matrix equations of the form

$$
V=\mathcal{Q}+\sum_{i=1}^{f} \mathscr{C}_{i} \varrho(V) \mathscr{C}_{i}^{*}-\sum_{j=1}^{k} \mathscr{D}_{j} \varrho(V) \mathscr{D}_{j}^{*}
$$


where $\mathcal{Q}$ is a positive definite matrix, $\mathscr{C}_{i}, \mathscr{D}_{j}$ are arbitrary $s \times s$ matrices for all $i=1, \ldots, f, j=1, \ldots, k$, and $\varrho$ is a self mapping on the set of all $s \times s$ Hermitian matrices, which maps the set of all $s \times s$ Hermitian positive definite matrices onto itself. To do this, we prove the existence of fixed points for multi-valued modified $F$-contractions in the frame of complete metric spaces. Henceforth, for a metric space $(U, m)$, define

$$
\begin{aligned}
2^{U} & =\{\mathscr{A} \subseteq U: \mathscr{A} \neq \varnothing\}, \\
C(U) & =\left\{\mathscr{A} \subseteq 2^{U}: \mathscr{A} \text { is closed }\right\}, \\
C B(U) & =\left\{\mathscr{A} \subseteq 2^{U}: \mathscr{A} \text { is closed and bounded }\right\}, \\
K(U) & =\left\{\mathscr{A} \subseteq 2^{U}: \mathscr{A} \text { is compact }\right\} .
\end{aligned}
$$

Note that $K(U) \subseteq C B(U) \subseteq C(U) \subseteq 2^{U}$. Let

$$
H(\mathscr{B}, \mathscr{A})=\max \left\{\sup _{\mu \in \mathscr{B}} M(\mu, \mathscr{A}), \sup _{\omega \in \mathscr{A}} M(\omega, \mathscr{B})\right\},
$$

where $M(\mu, \mathscr{B})=\inf \{m(\mu, \omega): \omega \in \mathscr{B}\}$ and $\mathscr{A}, \mathscr{B} \in C(U)$. Symbolize

$$
\mathfrak{F}=\left\{F: \mathbb{R}^{+} \rightarrow \mathbb{R}: F \text { satisfies }(F 1),(F 2) \text { and }(F 3)\right\}
$$

and

$$
\mathfrak{F}_{*}=\{F \in \mathfrak{F}: F \text { satisfies }(F 4)\},
$$

where

(F1) $\quad F$ is strictly increasing;

(F2) for all, sequence $\left\{r_{q}\right\} \subseteq R^{+}, \lim _{q \rightarrow \infty} r_{q}=0$ if and only if $\lim _{q \rightarrow \infty} F\left(r_{q}\right)=-\infty$;

(F3) there exist $0<k<1$ such that $\lim _{n \rightarrow 0^{+}} t^{k} F(t)=0$; and

(F4) $\quad F(\inf \mathscr{A})=\inf F(\mathscr{A})$ for all $\mathscr{A} \subset(0, \infty)$ with $\inf \mathscr{A}>0$.

Feng and Liu gave an important and interesting generalization of Nadler's fixed point theorem [5] as:

Theorem 1. [6] Let $(U, m)$ be a complete metric space and $\mathcal{G}: U \rightarrow C(U)$. If there exists $a, c \in(0,1)$ such that $c<a$ and for any $\mu \in U$, there is $\omega \in I_{a}^{\mu}$ satisfying

$$
M(\omega, \mathcal{G} \omega) \leq c m(\mu, \omega),
$$

where $I_{a}^{\mu}=\{\omega \in \mathcal{G} \mu: \operatorname{am}(\mu, \omega) \leq M(\mu, \mathcal{G} \mu)\}$. Then, $\mathcal{G}$ has a fixed point, provided that the map $\mu \rightarrow$ $M(\mu, \mathcal{G} \mu)$ is lower semi-continuous.

Altun et al. [7] defined multi-valued F-contractions and found some fixed point results. Further, Minak et al. [8] extended Theorem 1 and claimed that their obtained results are factual or proper generalizations of Feng and Liu's theorem (Theorem 1). However, Nguyen et al. [9] showed that their claim is not true by giving an example (see Example 1.1 in [9]) and gave refinements of Minak et al.'s theorems [9] by replacing "for any $\mu \in U$ there is $\omega \in \mathcal{G} \mu$ " by "for any $\mu \in U$ there is $\omega \in U$ " and extending functions $F$ to $[0, \infty)$ by putting $F(0)=-\infty$. Very recently, Nashine and Kadelburg [10] proved the following result as generalization of Theorem 1 . 
Theorem 2. Let $(U, m)$ be a complete metric space, $\mathcal{G}: U \rightarrow C(U)$ and $F \in \mathfrak{F}_{*}$ [10]. If there exist two functions $\xi:(0, \infty) \rightarrow(0, \infty)$ and $\pi:(0, \infty) \rightarrow(\xi, \infty)$ such that

$$
\pi(t)>\xi(t), \quad \lim _{t \rightarrow s^{+}} \inf \pi(t)>\lim _{t \rightarrow s^{+}} \inf \xi(t) \quad \text { for } s \geq 0
$$

and, for any $\mu \in U$ with $M(\mu, \mathcal{G} \mu)>0$, there exists $\omega \in \mathbb{F}_{\tilde{\mathcal{S}}}^{\mu}$ satisfying

$$
\pi\left(M_{\mu, \omega}\right)+F(M(\omega, \mathcal{G} \omega)) \leq F(m(\mu, \omega)),
$$

where $\mathbb{F}_{\xi}^{\mu}=\left\{\omega \in \mathcal{G} \mu: F(m(\mu, \omega)) \leq F(\max \{m(\mu, \mathcal{G} \mu), m(\omega, \mathcal{G} \omega)\})+\xi\left(M_{\mu, \omega}\right)\right\}$ and $M_{\mu, \omega}$ is defined in Equation (8), then $\mathcal{G}$ has a fixed point, provided that the map $\mu \rightarrow M(\mu, \mathcal{G} \mu)$ is lower semi-continuous.

However, the following example shows that Theorem 2 is not proper generalization of Theorem 1 .

Example 1. Let $U=[0,1] \subset \mathbb{R}$ with the usual metric $m$. Then, $(U, m)$ is complete metric space. Consider $\xi:(0, \infty) \rightarrow(0, \infty)$ and $\pi:(0, \infty) \rightarrow(\xi, \infty)$ are two functions satisfying Equation (5). Define $\mathcal{G}: U \rightarrow$ $C(U)$ by

$$
\mathcal{G} \mu= \begin{cases}U & \text { if } \mu=0 \\ \{0\} & \text { if } \mu \neq 0 .\end{cases}
$$

Then, $M(\mu, \mathcal{G} \mu)=\mu$ for all $x \in V$. In this example, Theorem 2 cannot be applied. Indeed, for $\mu \neq 0$, we have $M(\mu, \mathcal{G} \mu)>0$. Therefore, if $\omega \in \mathcal{G} \mu$, then $\omega=0$ and $M(\omega, \mathcal{G} \omega)=0$, thus Equation (6) is not satisfied for any $F$.

Motivated by Nguyen et al. [9], we overcome the error mentioned in Example 1.1 of [9] by another way. We define contractions involving $F$-functions and prove fixed point results for these type of contractions. In our results, the domain of the function $F$ is not extended from $(0, \infty)$ to $[0, \infty)$. Our results generalize (see [11-31]), refine, and extend the results of $[6,8,32,33]$.

\section{Fixed Point Results}

Let $\mathcal{G}: U \rightarrow 2^{U}$ be the multi-valued map, $F \in \mathfrak{F}, \xi:(0, \infty) \rightarrow(\xi, \infty), \xi>0$ and $\mu \in U$ with $M(\mu, \mathcal{G} \mu)>0$. Define the set

$$
\mathbb{F}_{\tilde{\xi}}^{\mu}=\left\{\omega \in \mathcal{G} \mu: M(\omega, \mathcal{G} \omega)>0 \text { and } F(m(\mu, \omega)) \leq F\left(M_{\mu, \omega}\right)+\xi(m(\mu, \omega))\right\},
$$

where

$$
M_{\mu, \omega}=\max \left\{m(\mu, \omega), M(\mu, \mathcal{G} \mu), M(\omega, \mathcal{G} \omega), \frac{M(\omega, \mathcal{G} \mu)+M(\mu, \mathcal{G} \omega)}{2}\right\}
$$

By considering $\xi:(0, \infty) \rightarrow(\xi, \infty), \xi>0$ a constant function, that is, $\xi(t)=\xi+$ constant $=b$, Equation (7) becomes

$$
\mathbb{F}_{b}^{\mu}=\{\omega \in \mathcal{G} \mu: M(\omega, \mathcal{G} \omega)>0 \text { and } F(m(\mu, \omega)) \leq F(M(\mu, \mathcal{G} \mu))+b\} .
$$

Definition 1. Let $\mathcal{G}: U \rightarrow 2^{U}$ be a multi-valued mapping on a metric space $(U, m)$; then, $\mathcal{G}$ is said to be modified-F-contraction on $U$, if there exists $\pi:(0, \infty) \rightarrow(0, \infty), \xi:(0, \infty) \rightarrow(\xi, \infty), \xi>0$ and a function $F \in \mathfrak{F}$ such that, for all $\mu \in U$ with $M(\mu, \mathcal{G} \mu)>0$, there exists $\omega \in \mathbb{F}_{\tilde{\xi}}^{\mu}$ satisfying

$$
\pi(m(\mu, \omega))+F(M(\omega, \mathcal{G} \omega)) \leq F\left(M_{\mu, \omega}\right),
$$

where $M_{\mu, \omega}$ is defined in Equation (8). 
Now, we prove our main results.

Theorem 3. Let $(U, m)$ be a complete metric space and $\mathcal{G}: U \rightarrow K(U)$ be a multi-valued mapping satisfying the following assertions:

1. $\mathcal{G}$ is modified-F-contraction for $F \in \mathfrak{F}$;

2. $\quad \mu \rightarrow M(\mu, \mathcal{G} \mu)$ is lower semi-continuous mapping; and

3. $\pi:(0, \infty) \rightarrow(0, \infty)$ and $\xi:(0, \infty) \rightarrow(\xi, \infty), \xi>0$ satisfy

$$
\pi(t)>\xi(t)
$$

and

$$
\lim _{t \rightarrow s^{+}} \inf \pi(t)>\lim _{t \rightarrow s^{+}} \inf \xi(t) \quad \text { for all } \quad s \geq 0
$$

Then, $\mathcal{G}$ has a fixed point in $U$.

Proof. Assume that $\mathcal{G}$ has no fixed point in $U$. Let $\mu_{0} \in U$. Then, $M\left(\mu_{0}, \mathcal{G} \mu_{0}\right)>0$, otherwise $\mu_{0}$ is the fixed point of $\mathcal{G}$. Since $\mathcal{G} \mu \in K(U)$ for every $\mu$, there exists $\mu_{1} \in \mathcal{G} \mu_{0}$ such that $m\left(\mu_{0}, \mu_{1}\right)=M\left(\mu_{0}, \mathcal{G} \mu_{0}\right)$. It also follows that

$$
F\left(m\left(\mu_{0}, \mu_{1}\right)\right) \leq F\left(M_{\mu_{0}, \mu_{1}}\right)+\xi\left(m\left(\mu_{0}, \mu_{1}\right)\right)
$$

$M\left(\mu_{1}, \mathcal{G} \mu_{1}\right)>0$, otherwise $\mu_{1}$ is the fixed point of $\mathcal{G}$. Thus, $\mu_{1} \in \mathbb{F}_{\tilde{\xi}}^{\mu_{0}}$ and $\mu_{1} \neq \mu_{0}$, therefore, from Equation (10), we have

$$
\pi\left(m\left(\mu_{0}, \mu_{1}\right)\right)+F\left(M\left(\mu_{1}, \mathcal{G} \mu_{1}\right)\right) \leq F\left(M_{\mu_{0}, \mu_{1}}\right),
$$

where

$$
\begin{aligned}
& M_{\mu_{0, \mu_{1}}}=\max \left\{m\left(\mu_{0}, \mu_{1}\right), M\left(\mu_{0}, \mathcal{G} \mu_{0}\right), M\left(\mu_{1}, \mathcal{G} \mu_{1}\right),\right. \\
& \left.\frac{M\left(\mu_{1}, \mathcal{G} \mu_{0}\right)+M\left(\mu_{0}, \mathcal{G} \mu_{1}\right)}{2}\right\} .
\end{aligned}
$$

Since $\mathcal{G} \mu_{0}$ and $\mathcal{G} \mu_{1}$ are compact, Equation (15) gives

$$
M_{\mu_{0}, \mu_{1}}=\max \left\{m\left(\mu_{0}, \mu_{1}\right), m\left(\mu_{1}, \mu_{2}\right), \frac{m\left(\mu_{0}, \mu_{2}\right)}{2}\right\} .
$$

Since

$$
\begin{aligned}
\frac{m\left(\mu_{0}, \mu_{2}\right)}{2} & \leq \frac{m\left(\mu_{0}, \mu_{1}\right)+m\left(\mu_{1}, \mu_{2}\right)}{2} \\
& \leq \max \left\{m\left(\mu_{0}, \mu_{1}\right), m\left(\mu_{1}, \mu_{2}\right)\right\},
\end{aligned}
$$

it follows that

$$
M_{\mu_{0}, \mu_{1}} \leq \max \left\{m\left(\mu_{0}, \mu_{1}\right), m\left(\mu_{1}, \mu_{2}\right)\right\} .
$$

Suppose that $m\left(\mu_{0}, \mu_{1}\right)<m\left(\mu_{1}, \mu_{2}\right)$; then, Equation (14) implies that

$$
\pi\left(m\left(\mu_{0}, \mu_{1}\right)\right)+F\left(M\left(\mu_{1}, \mathcal{G} \mu_{1}\right)\right) \leq F\left(m\left(\mu_{1}, \mu_{2}\right)\right) ;
$$


consequently,

$$
\pi\left(m\left(\mu_{0}, \mu_{1}\right)\right)+F\left(m\left(\mu_{1}, \mu_{2}\right)\right) \leq\left(m\left(\mu_{1}, \mu_{2}\right)\right),
$$

or $F\left(m\left(\mu_{1}, \mu_{2}\right)\right) \leq F\left(m\left(\mu_{1}, \mu_{2}\right)\right)-\pi\left(m\left(\mu_{0}, \mu_{1}\right)\right)$, which is a contradiction. Hence, $M_{\mu_{0}, \mu_{1}} \leq m\left(\mu_{0}, \mu_{1}\right)$; therefore, by using (F1), Equations (13) and (14) imply that

$$
F\left(m\left(\mu_{0}, \mu_{1}\right)\right) \leq F\left(m\left(\mu_{0}, \mu_{1}\right)\right)+\xi\left(m\left(\mu_{0}, \mu_{1}\right)\right)
$$

and

$$
\pi\left(m\left(\mu_{0}, \mu_{1}\right)\right)+F\left(m\left(\mu_{1}, \mu_{2}\right) \leq F\left(m\left(\mu_{0}, \mu_{1}\right)\right) .\right.
$$

On continuing recursively, we get a sequence $\left\{\mu_{q}: \mu_{q} \in \mathcal{G} \mu_{q-1}\right\}_{n \in \mathbb{N}}$ in $U$, where $\mu_{q+1} \in \mathbb{F}_{\tilde{\xi}}^{\mu_{q}}, \mu_{q+1} \notin$ $\mathcal{G} \mu_{q+1}, M_{\mu_{q}, \mu_{q+1}} \leq m\left(\mu_{q}, \mu_{q+1}\right)$ and

$$
\pi\left(m\left(\mu_{q}, \mu_{q+1}\right)\right)+F\left(M\left(\mu_{q+1}, \mathcal{G} \mu_{q+1}\right)\right) \leq F\left(m\left(\mu_{q}, \mu_{q+1}\right)\right) .
$$

Since $\mu_{q+1} \in \mathbb{F}_{\tilde{\xi}}^{\mu_{q}}$ and $\mathcal{G} \mu_{q}$ and $\mathcal{G} \mu_{q+1}$ are compact, we have

$$
\pi\left(m\left(\mu_{q}, \mu_{q+1}\right)\right)+F\left(m\left(\mu_{q+1}, \mu_{q+2}\right)\right) \leq F\left(m\left(\mu_{q}, \mu_{q+1}\right)\right)
$$

and

$$
F\left(m\left(\mu_{q}, \mu_{q+1}\right)\right) \leq F\left(m\left(\mu_{q}, \mu_{q+1}\right)\right)+\xi\left(m\left(\mu_{q}, \mu_{q+1}\right)\right) .
$$

Combining Equations (23) and (24) gives

$$
\begin{aligned}
F\left(m\left(\mu_{q+1}, \mu_{n+2}\right)\right) & \leq F\left(m\left(\mu_{q}, \mu_{q+1}\right)\right)+\xi\left(m\left(\mu_{q}, \mu_{q+1}\right)\right) \\
& -\pi\left(m\left(\mu_{q}, \mu_{q+1}\right)\right)
\end{aligned}
$$

Set $m\left(\mu_{q}, \mu_{q+1}\right)=L_{q}$. From Equation (25), we get

$$
\begin{aligned}
F\left(L_{q+1}\right) & \leq F\left(L_{q}\right)+\xi\left(L_{q}\right)-\pi\left(L_{q}\right) \\
& \leq F\left(L_{q-1}\right)+\xi\left(L_{q}\right)+\xi\left(L_{q-1}\right)-\pi\left(L_{q}\right)-\pi\left(L_{q-1}\right) \\
& \vdots \\
& \leq F\left(L_{0}\right)+\xi\left(L_{q}\right)+\xi\left(L_{q-1}\right)+\cdots+\xi\left(L_{0}\right) \\
& -\pi\left(L_{q}\right)-\pi\left(L_{q-1}\right)-\cdots-\pi\left(L_{0}\right) .
\end{aligned}
$$

Let $\pi\left(L_{p_{n}}\right)=\min \left\{\pi\left(L_{0}\right), \pi\left(L_{1}\right), \cdots, \pi\left(L_{q}\right)\right\}$ and $\xi\left(L_{q_{n}}\right)=\max \left\{\xi\left(L_{0}\right), \xi\left(L_{1}\right), \cdots, \xi\left(L_{q}\right)\right\}$ for all $n \in$ $\mathbb{N}$. From Equation (26), we get

$$
F\left(L_{q+1}\right) \leq F\left(L_{0}\right)+q\left(\xi\left(L_{q_{n}}\right)-\pi\left(L_{p_{n}}\right)\right) .
$$

From Equation (22), we also get

$$
\begin{array}{r}
F\left(M\left(\mu_{q+1}, \mathcal{G} \mu_{q+1}\right)\right) \leq F\left(M\left(\mu_{0}, \mathcal{G} \mu_{0}\right)\right)+ \\
n\left(\xi\left(L_{q_{n}}\right)-\pi\left(L_{p_{n}}\right)\right) .
\end{array}
$$


Equations (12) and (28) imply $\lim _{q \rightarrow \infty} F\left(L_{q}\right)=-\infty$, thus, by $(F 2), \lim _{q \rightarrow \infty} L_{q}=0$. Now, we prove that $\left\{\mu_{q}: \mu_{q} \in \mathcal{G} \mu_{q-1}\right\}$ is a Cauchy sequence. From (F3), there exists $0<r<1$ such that

$$
\lim _{q \rightarrow \infty}\left(L_{q}\right)^{r} F\left(L_{q}\right)=0 .
$$

By Equation (27), we get for all $n \in \mathbb{N}$

$$
\left(L_{q}\right)^{r} F\left(L_{q}\right)-\left(L_{q}\right)^{r} F\left(L_{0}\right) \leq\left(L_{q}\right)^{r} q\left(\xi\left(L_{q_{n}}\right)-\pi\left(L_{p_{n}}\right)\right) \leq 0 .
$$

Letting $q \rightarrow \infty$ in Equation (30), we obtain

$$
\lim _{q \rightarrow \infty} q\left(L_{q}\right)^{r}=0
$$

This implies that there exists $n_{1} \in \mathbb{N}$ such that $q\left(L_{q}\right)^{r} \leq 1$ or $L_{q} \leq \frac{1}{q^{1 / r}}$, for all $q>q_{1}$. Next, for $m>q \geq q_{1}$, we have

$$
m\left(\mu_{q}, \mu_{m}\right) \leq \sum_{i=q}^{f-1} m\left(\mu_{i}, \mu_{i+1}\right) \leq \sum_{l=q}^{f-1} \frac{1}{l^{1 / k}},
$$

since $0<k<1, \sum_{l=q}^{f-1} \frac{1}{l^{1 / k}}$ converges. Therefore, $m\left(\mu_{q}, \mu_{f}\right) \rightarrow 0$ as $f, q \rightarrow \infty$. Thus, $\left\{\mu_{q}: \mu_{q} \in \mathcal{G} \mu_{q-1}\right\}$ is a Cauchy sequence. Since $U$ is complete, there exists $\mu^{*} \in U$ such that $\mu_{q} \rightarrow \mu^{*}$ as $q \rightarrow \infty$. From Equation (28) and (F2), we have

$$
\lim _{q \rightarrow \infty} M\left(\mu_{q}, \mathcal{G} \mu_{q}\right)=0
$$

From the hypothesis in Equation (2), we obtain

$$
0 \leq M(\mu, \mathcal{G} \mu) \leq \lim _{q \rightarrow \infty} \inf M\left(\mu_{q}, \mathcal{G} \mu_{q}\right)=0,
$$

which is a contradiction. Thus, $\mathcal{G}$ has a fixed point.

In the following theorem, we take $C(U)$ instead of $K(U)$; thus, we need to take $F \in \mathfrak{F}_{*}$.

Theorem 4. Let $(U, m)$ be a complete metric space and $\mathcal{G}: U \rightarrow C(U)$ be a multi-valued mapping such that $\mathcal{G}$ is modified-F-contraction for $F \in \mathfrak{F}_{*}$ and satisfying the assertions in Equations (2) and (3) of Theorem 3. Then, $\mathcal{G}$ has a fixed point in $U$.

Proof. Assume that $\mathcal{G}$ has no fixed point in $U$. Let $\mu_{0} \in U$, then $M\left(\mu_{0}, \mathcal{G} \mu_{0}\right)>0$, otherwise $\mu_{0}$ is the fixed point of $\mathcal{G}$. Since $\mathcal{G} \mu \in C(U)$ for every $\mu \in U$ and $F \in \mathfrak{F}_{*}$, there exist $\mu_{1} \in \mathcal{G} \mu_{0}$ such that

$$
\begin{aligned}
F\left(m\left(\mu_{0}, \mu_{1}\right)\right) & \leq \inf \left\{F\left(m\left(\mu_{0}, \mu_{1}\right)\right): \mu_{1} \in \mathcal{G} \mu_{0}\right\}+\xi\left(m\left(\mu_{0}, \mu_{1}\right)\right. \\
& =F\left(\inf \left\{m\left(\mu_{0}, \mu_{1}\right): \mu_{1} \in \mathcal{G} \mu_{0}\right\}\right)+\xi\left(m\left(\mu_{0}, \mu_{1}\right)\right. \\
& =F\left(M\left(\mu_{0}, \mathcal{G} \mu_{0}\right)\right)+\xi\left(m\left(\mu_{0}, \mu_{1}\right)\right. \\
& \leq F\left(M_{\mu_{0}, \mu_{1}}\right)+\xi\left(m\left(\mu_{0}, \mu_{1}\right)\right.
\end{aligned}
$$

and $M\left(\mu_{1}, \mathcal{G} \mu_{1}\right)>0$, otherwise $\mu_{1}$ is the fixed point of $\mathcal{G}$. Thus, from Equation (10), we have

$$
\pi\left(m\left(\mu_{0}, \mu_{1}\right)\right)+F\left(M\left(\mu_{1}, \mathcal{G} \mu_{1}\right)\right) \leq F\left(M_{\mu_{0}, \mu_{1}}\right),
$$


where

$$
\begin{aligned}
M_{\mu_{0}, \mu_{1}} & =\max \left\{m\left(\mu_{0}, \mu_{1}\right), M\left(\mu_{0}, \mathcal{G} \mu_{0}\right), M\left(\mu_{1}, \mathcal{G} \mu_{1}\right),\right. \\
& \left.\frac{M\left(\mu_{1}, \mathcal{G} \mu_{0}\right)+M\left(\mu_{0}, \mathcal{G} \mu_{1}\right)}{2}\right\} \\
& \leq \max \left\{m\left(\mu_{0}, \mu_{1}\right), m\left(\mu_{0}, \mu_{1}\right), m\left(\mu_{1}, \mu_{2}\right),\right. \\
& \left.\frac{m\left(\mu_{1}, \mu_{1}\right)+m\left(\mu_{0}, \mu_{2}\right)}{2}\right\} .
\end{aligned}
$$

Since

$$
\begin{aligned}
\frac{m\left(\mu_{0}, \mu_{2}\right)}{2} & \leq \frac{m\left(\mu_{0}, \mu_{1}\right)+m\left(\mu_{1}, \mu_{2}\right)}{2} \\
& \leq \max \left\{m\left(\mu_{0}, \mu_{1}\right), m\left(\mu_{1}, \mu_{2}\right)\right\}
\end{aligned}
$$

it follows that

$$
M_{\mu_{0}, \mu_{1}} \leq \max \left\{m\left(\mu_{0}, \mu_{1}\right), m\left(\mu_{1}, \mu_{2}\right)\right\}
$$

Due to $(F 4)$, we obtain

$$
F\left(M\left(\mu_{1}, \mathcal{G} \mu_{1}\right)\right)=\inf _{\omega \in \mathcal{G} \mu} F\left(m\left(\mu_{1}, \omega\right)\right)
$$

Suppose that $m\left(\mu_{0}, \mu_{1}\right)<m\left(\mu_{1}, \mu_{2}\right)$; then, Equations (32) and (34) imply that

$$
\inf _{\omega \in \mathcal{G} \mu} F\left(m\left(\mu_{1}, \omega\right)\right) \leq F\left(m\left(\mu_{1}, \mu_{2}\right)\right)-\pi\left(m\left(\mu_{0}, \mu_{1}\right)\right) .
$$

Then, by Equation (35), there exists $\mu_{2} \in \mathcal{G} \mu_{1}$ such that

$$
F\left(m\left(\mu_{1}, \mu_{2}\right)\right) \leq F\left(m\left(\mu_{1}, \mu_{2}\right)\right)-\pi\left(m\left(\mu_{0}, \mu_{1}\right)\right),
$$

which is a contradiction. Hence, $M_{\mu_{0}, \mu_{1}} \leq m\left(\mu_{0}, \mu_{1}\right)$. Therefore, from Equations (32) and (34), we obtain

$$
F\left(m\left(\mu_{1}, \mu_{2}\right)\right) \leq F\left(m\left(\mu_{0}, \mu_{1}\right)\right)-\pi\left(m\left(\mu_{0}, \mu_{1}\right)\right),
$$

The rest of the proof can be completed as in the proof of Theorem 3.

By defining $\xi:(0, \infty) \rightarrow(\xi, \infty), \xi>0$ as $\xi(t)=\xi+$ constant $=b>0$ for all $t \in[0, \infty)$, we get

Corollary 1. Let $(U, m)$ be a complete metric space and $\mathcal{G}: U \rightarrow K(U)$ be a multi-valued mapping. If there exists $b>0$ and a function $\pi:(0, \infty) \rightarrow(b, \infty)$ such that

$$
\lim _{t \rightarrow s^{+}} \inf \pi(t)>b \quad \text { for } s \geq 0
$$

and for $F \in \mathfrak{F}, \mu \in U$ with $M(\mu, \mathcal{G} \mu)>0$, there exists $\omega \in \mathbb{F}_{b}^{\mu}$ satisfying

$$
\pi(m(\mu, \omega))+F(M(\omega, \mathcal{G} \omega)) \leq F\left(M_{\mu, \omega}\right) .
$$

Then, $\mathcal{G}$ has a fixed point in $U$, provided that $\mu \rightarrow M(\mu, \mathcal{G} \mu)$ is a lower semi-continuous mapping.

Corollary 2. Let $(U, m)$ be a complete metric space and $\mathcal{G}: U \rightarrow C(U)$ be a multi-valued mapping satisfying all the assertions of Corollary 1 for $F \in \mathfrak{F}_{*}$. Then, $\mathcal{G}$ has a fixed point in $U$. 
Corollary 3. Let $(U, m)$ be a complete metric space and $\mathcal{G}: U \rightarrow K(U)$ be a multi-valued mapping. If there exists $b>0$ and a function $\pi:(0, \infty) \rightarrow(b, \infty)$ such that

$$
\lim _{t \rightarrow s^{+}} \inf \pi(t)>b \quad \text { for } s \geq 0
$$

and for $F \in \mathfrak{F}, x \in U$ with $M(\mu, \mathcal{G} \mu)>0$, there exists $y \in \mathbb{F}_{b}^{x}$ satisfying at least one of the following:

$\left(\mathrm{F}_{1}\right) \quad \pi(m(\mu, \omega))+F(M(\omega, \mathcal{G} \omega)) \leq F(m(\mu, \omega)) ;$

$\left(\mathrm{F}_{2}\right) \quad \pi(m(\mu, \omega))+F(M(\omega, \mathcal{G} \omega)) \leq F(\max \{M(\mu, \mathcal{G} \mu), M(\omega, \mathcal{G} \omega)\}) ;$ and

$\left(\mathrm{F}_{3}\right) \quad \pi(m(\mu, \omega))+F(M(\omega, \mathcal{G} \omega)) \leq F\left(\frac{1}{2}[M(\mu, \mathcal{G} \omega)+M(\omega, \mathcal{G} \mu)]\right)$.

Then, $\mathcal{G}$ has a fixed point in $U$ provided that the map $\mu \rightarrow M(\mu, \mathcal{G} \mu)$ is lower semi-continuous.

Corollary 4. Let $(U, m)$ be a complete metric space and $\mathcal{G}: U \rightarrow C(U)$ be a multi-valued mapping satisfying all the assumptions of Corollary 3 for $F \in \mathfrak{F}_{*}$. Then, $\mathcal{G}$ has a fixed point in $U$.

Corollary 5. Let $(U, m)$ be a complete metric space and $\mathcal{G}: U \rightarrow C(U)$ be a multi-valued mapping. If there exists a function $\varphi:[0, \infty) \rightarrow(0,1)$ and a non-decreasing function $b:[0, \infty) \rightarrow[b, 1), b>0$ such that

$$
\varphi(t)<b(t) \text { and } \lim _{t \rightarrow s^{+}} \sup \varphi(t)<\lim _{t \rightarrow s^{+}} \sup b(t)
$$

for all $t \in[0, \infty)$ and for any $\mu \in U$ there is $\omega \in \mathcal{G} \mu$ satisfying the following two conditions:

$$
b(m(\mu, \omega)) m(\mu, \omega) \leq M_{\mu, \omega}
$$

and

$$
M(\omega, T \omega) \leq \varphi(m(\mu, \omega)) M_{\mu, \omega}
$$

where $M_{\mu, \omega}$ is defined in Equation (8). Then, $\mathcal{G}$ has a fixed point in $U$ provided that $\mu \rightarrow M(\mu, \mathcal{G} \mu)$ is lower semi-continuous.

Proof. Define $F:[0, \infty) \rightarrow \mathbb{R}, \pi:(0, \infty) \rightarrow(0, \infty)$ and $\xi:(0, \infty) \rightarrow(\xi, \infty), \xi>0$ by $F(r)=\ln r$ for $r \in(0,1), \pi(t)=-\ln \varphi(t)$ and $\xi(t)=-\ln b(t)$ for $t \in(0, \infty)$. Then, all conditions of Theorem 4 hold true and thus $\mathcal{G}$ has a fixed point in $U$.

Corollary 6. Let $(U, m)$ be a complete metric space and $\mathcal{G}: U \rightarrow K(U)$ be a multi-valued mapping satisfying all the assertions of Corollary 5 for $F \in \mathfrak{F}$. Then, $\mathcal{G}$ has a fixed point in $U$.

Corollary 7. Let $(U, m)$ be a complete metric space and $\mathcal{G}: U \rightarrow C(U)$ be a multi-valued mapping. If there exists constants $b, c \in(0,1)$ such that $c<b$ and for any $\mu \in U$ there is $\omega \in T x$ satisfying the following conditions:

$$
b d(\mu, \omega) \leq M_{\mu, \omega}
$$

and

$$
M(\omega, \mathcal{G} \omega) \leq c M_{\mu, \omega}
$$

where $M_{\mu, \omega}$ is defined in Equation (8). Then, $\mathcal{G}$ has a fixed point in $U$ provided that $\mu \rightarrow M(\mu, \mathcal{G} \mu)$ is lower semi-continuous. 
Proof. Define $\varphi:[0, \infty) \rightarrow(0,1)$ and $b:[0, \infty) \rightarrow[b, 1)$ by $\varphi(t)=c$ and $b(t)=b$, respectively, for all $t \in[0, \infty)$, where $b, c \in(0,1)$. Then, all conditions of Corollary 5 are satisfied and hence $\mathcal{G}$ has a fixed point.

Remark 1. Corollary 5 generalizes Theorem 6 of [33] and Corollary 7 generalizes the Theorem 1.

Example 2. Let $U=\{0,1,2,3, \ldots\}$ with

$$
m(\mu, \omega)= \begin{cases}0 & \text { if } \mu=\omega, \\ \mu+\omega & \text { if } \mu \neq \omega,\end{cases}
$$

then $(U, m)$ is complete metric space. Define $\mathcal{G}: U \rightarrow C(U), F: \mathbb{R}^{+} \rightarrow \mathbb{R}, \pi:(0, \infty) \rightarrow(0, \infty)$ and $\xi:(0, \infty) \rightarrow(\xi, \infty)$ by

$$
\mathcal{G} \mu= \begin{cases}\{0,4\} & \text { if } \mu \in\{0,3\} \\ \{2,3\} & \text { if } \mu \in\{2\} \\ \{1,2, \ldots, \mu-1\} & \text { if } \mu \in\{4,5, \ldots\} \cup\{1\}\end{cases}
$$

$F(t)=\ln (t), \pi(t)=\frac{1}{t}+0.12$, and $\xi(t)=\frac{1}{t}+0.05$ for all $t>0$. Then,

$$
M(\mu, \mathcal{G} \mu)=\left\{\begin{array}{cc}
0 & \text { if } \mu \in\{0,1,2\} \\
3 & \text { if } \mu=3 \\
\mu+1 & \text { if } \mu \in\{4,5, \ldots\}
\end{array}\right.
$$

$\pi(t)>\xi(t)$ and $\lim _{t \rightarrow s^{+}} \inf \pi(t)>\lim _{t \rightarrow s^{+}} \inf \xi(t)$ for all $s \geq 0$. Now, let $M(\mu, \mathcal{G} \mu)>0$; then, there exists two cases:

Case 1. When $\mu=3, \mathcal{G} \mu=\{0,4\}$. Thus, for $\omega=4 \in \mathcal{G} \mu$ such that $M(4, \mathcal{G} 4)=5>0$, we have

$$
F(m(\mu, \omega))-F\left(M_{\mu, \omega}\right)=F(7)-F(7)=0<\xi(m(\mu, \omega)) .
$$

In addition,

$$
\begin{aligned}
F(M(\omega, \mathcal{G} \omega))-F\left(M_{\mu, \omega}\right) & =F(5)-F(7) \\
& =\ln \left(\frac{5}{7}\right) \\
& =-0.336 \\
& <-\left(\frac{1}{7}+0.12\right) \\
& =-\pi(m(\mu, \omega)) .
\end{aligned}
$$

Case 2. When $\mu \in\{4,5, \ldots\}$,

$\mathcal{G} \mu=\{1,2, \ldots, \mu-1\}$. Thus, for $\omega=3 \in \mathcal{G} \mu$ such that $M(3, \mathcal{G} 3)=3>0$, we have

$$
\begin{aligned}
F(m(\mu, \omega))-F\left(M_{\mu, \omega}\right) & =F(\mu+1)-F(\mu+1) \\
& =0<\xi(m(\mu, \omega)) .
\end{aligned}
$$


In addition,

$$
\begin{aligned}
F(M(\omega, \mathcal{G} \omega))-F\left(M_{\mu, \omega}\right) & =F(3)-F(\mu+1) \\
& =\ln \left(\frac{3}{\mu+1}\right) \\
& \leq-\left(\frac{1}{\mu+3}+0.12\right) \\
& =-\pi(m(\mu, \omega)) .
\end{aligned}
$$

Hence, $\mathcal{G}$ is modified-F-contraction.

Next, let $\lim _{q \rightarrow \infty} m\left(\mu_{q}, \mu\right)=0$. Then,

$$
\lim _{q \rightarrow \infty} \inf M\left(\mu_{q}, \mathcal{G} \mu_{q}\right)=M(\mu, \mathcal{G} \mu) .
$$

Hence, $\mathcal{G}$ is a lower semi-continuous mapping. Thus, all conditions of Theorem 4 hold and 0,1 , and 2 are fixed points of $\mathcal{G}$.

Remark 2. In Example 2, Theorem 2 cannot be applied. Indeed, for $\mu=3, \mathcal{G} \mu=\{0,4\}$. Thus, for $\omega=4 \in \mathcal{G} \mu$ such that $M(4, \mathcal{G} 4)=5>0$, we have

$$
\begin{aligned}
& F(m(\mu, \omega))-F(\max \{M(\mu, \mathcal{G} \mu), M(\omega, \mathcal{G} \omega)\}) \\
= & F(7)-F(5) \\
= & \ln \left(\frac{7}{5}\right) \\
< & \xi(m(\mu, \omega)) .
\end{aligned}
$$

Then,

$$
\begin{aligned}
& F(M(\omega, \mathcal{G} \omega))-F(m(\mu, \omega)) \\
= & F(5)-F(7) \\
= & -\ln \left(\frac{7}{5}\right) \\
\geq & -\xi(m(\mu, \omega)) \\
\geq & -\pi\left(M_{\mu, \omega}\right) .
\end{aligned}
$$

Hence, Equation (6) does not hold.

Definition 2. Let $\mathcal{G}: U \rightarrow 2^{U}$ be a multi-valued mapping on a metric space $(U, m), F_{1}:(0, \infty) \rightarrow \mathbb{R}$ be a nondecreasing function, and $F_{2}:(0, \infty) \rightarrow \mathbb{R}$ satisfy $(F 2)$ and $(F 3)$. Then, $\mathcal{G}$ is said to be $F_{1}-F_{2}$-contraction on $U$, if there exists $\pi:(0, \infty) \rightarrow(0, \infty)$ and $\xi:(0, \infty) \rightarrow(\xi, \infty), \xi>0$ such that, for all $x \in U$ with $M(\mu, \mathcal{G} \mu)>0$, there exists $\omega \in \mathcal{G} \mu$ with $M(\omega, \mathcal{G} \omega)>0$ satisfying

$$
\pi(m(\mu, \omega))+F_{1}(M(\omega, \mathcal{G} \omega)) \leq F_{1}\left(M_{\mu, \omega}\right)
$$

and

$$
F_{2}(m(\mu, \omega)) \leq F_{1}(M(\mu, \mathcal{G} \mu))+\xi(m(\mu, \omega)),
$$

where $M_{1}(\mu, \omega)$ is defined in Equation (8).

Theorem 5. Let $(U, m)$ be a complete metric space and $\mathcal{G}: U \rightarrow K(U)$ be a $F_{1}-F_{2}$-contraction satisfying the hypotheses in Equations (2) and (3) of Theorem 3. Then, $\mathcal{G}$ has a fixed point in $U$. 
Proof. Assume that $\mathcal{G}$ has no fixed point in $U$. Let $\mu_{0} \in U$; then, $M\left(\mu_{0}, \mathcal{G} \mu_{0}\right)>0$, otherwise $\mu_{0}$ is the fixed point of $\mathcal{G}$. Since $\mathcal{G} \mu \in K(U)$ for every $\mu$, there exists $\mu_{1} \in \mathcal{G} \mu_{0}$ such that $m\left(\mu_{0}, \mu_{1}\right)=M\left(\mu_{0}, \mathcal{G} \mu_{0}\right)$ with $M\left(\mu_{1}, \mathcal{G} \mu_{1}\right)>0$, otherwise $\mu_{1}$ is the fixed point of $\mathcal{G}$. Thus, from Equations (38) and (39), we have

$$
\pi\left(m\left(\mu_{0}, \mu_{1}\right)\right)+F_{1}\left(M\left(\mu_{1}, \mathcal{G} \mu_{1}\right)\right) \leq F_{1}\left(M_{\mu_{0}, \mu_{1}}\right),
$$

and

$$
F_{2}\left(m\left(\mu_{0}, \mu_{1}\right)\right) \leq F_{1}\left(M\left(\mu_{0}, \mathcal{G} \mu_{0}\right)\right)+\xi\left(m\left(\mu_{0}, \mu_{1}\right)\right)
$$

where

$$
\begin{aligned}
M_{\mu_{0}, \mu_{1}}= & \max \left\{m\left(\mu_{0}, \mu_{1}\right), M\left(\mu_{0}, \mathcal{G} \mu_{0}\right), M\left(\mu_{1}, \mathcal{G} \mu_{1}\right),\right. \\
& \left.\frac{M\left(\mu_{1}, \mathcal{G} \mu_{0}\right)+M\left(\mu_{0}, \mathcal{G} \mu_{1}\right)}{2}\right\} .
\end{aligned}
$$

Since $\mathcal{G} \mu_{0}$ and $\mathcal{G} \mu_{1}$ are compact, Equation (42) gives

$$
\begin{aligned}
M_{\mu_{0}, \mu_{1}}= & \max \left\{m\left(\mu_{0}, \mu_{1}\right), m\left(\mu_{0}, \mu_{1}\right), m\left(\mu_{1}, \mu_{2}\right),\right. \\
& \left.\frac{m\left(\mu_{1}, \mu_{1}\right)+m\left(\mu_{0}, \mu_{2}\right)}{2}\right\} \\
= & \max \left\{m\left(\mu_{0}, \mu_{1}\right), m\left(\mu_{1}, \mu_{2}\right), \frac{m\left(\mu_{0}, \mu_{2}\right)}{2}\right\} .
\end{aligned}
$$

Since

$$
\begin{aligned}
\frac{m\left(\mu_{0}, \mu_{2}\right)}{2} & \leq \frac{m\left(\mu_{0}, \mu_{1}\right)+m\left(\mu_{1}, \mu_{2}\right)}{2} \\
& \leq \max \left\{m\left(\mu_{0}, \mu_{1}\right), m\left(\mu_{1}, \mu_{2}\right)\right\},
\end{aligned}
$$

it follows that

$$
M_{\mu_{0}, \mu_{1}} \leq \max \left\{m\left(\mu_{0}, \mu_{1}\right), m\left(\mu_{1}, \mu_{2}\right)\right\} .
$$

Suppose that $m\left(\mu_{0}, \mu_{1}\right)<m\left(\mu_{1}, \mu_{2}\right)$; then, Equation (40) implies that

$$
\pi\left(m\left(\mu_{0}, \mu_{1}\right)\right)+F_{1}\left(M\left(\mu_{1}, \mathcal{G} \mu_{1}\right)\right) \leq F_{1}\left(m\left(\mu_{1}, \mu_{2}\right)\right) ;
$$

consequently,

$$
\pi\left(m\left(\mu_{0}, \mu_{1}\right)\right)+F_{1}\left(m\left(\mu_{1}, \mu_{2}\right)\right) \leq F_{1}\left(m\left(\mu_{1}, \mu_{2}\right)\right),
$$

or $F\left(m\left(\mu_{1}, \mu_{2}\right)\right) \leq F\left(m\left(\mu_{1}, \mu_{2}\right)\right)-\pi\left(m\left(\mu_{0}, \mu_{1}\right)\right)$, which is a contradiction. Hence, $M_{\mu_{0}, \mu_{1}} \leq m\left(\mu_{0}, \mu_{1}\right)$. Since $F_{1}$ is nondecreasing, from Equation (40), we get

$$
\pi\left(m\left(\mu_{0}, \mu_{1}\right)\right)+F_{1}\left(m\left(\mu_{1}, \mu_{2}\right) \leq F_{1}\left(m\left(\mu_{0}, \mu_{1}\right)\right) .\right.
$$

On continuing recursively, we get a sequence $\left\{\mu_{q}: \mu_{q} \in \mathcal{G} \mu_{q-1}\right\}_{n \in \mathbb{N}}$ in $U$, where $M\left(\mu_{q}, \mathcal{G} \mu_{q}\right)>0$, $\mu_{q+1} \notin \mathcal{G} \mu_{q+1}$ and $M_{\mu_{q}, \mu_{q+1}} \leq m\left(\mu_{q}, \mu_{q+1}\right)$ satisfying

$$
\pi\left(m\left(\mu_{q}, \mu_{q+1}\right)\right)+F_{1}\left(M\left(\mu_{q+1}, \mathcal{G} \mu_{q+1}\right)\right) \leq F_{1}\left(m\left(\mu_{q}, \mu_{q+1}\right)\right) .
$$


and

$$
F_{2}\left(m\left(\mu_{q}, \mu_{q+1}\right)\right) \leq F_{1}\left(m\left(\mu_{q}, \mu_{q+1}\right)\right)+\xi\left(m\left(\mu_{q}, \mu_{q+1}\right)\right)
$$

Since $\mu_{q+1} \in \mathbb{F}_{1,2}^{\xi, \mu_{q}}$ and $\mathcal{G} \mu_{q}$ and $\mathcal{G} \mu_{q+1}$ are compact, we have

$$
\pi\left(m\left(\mu_{q}, \mu_{q+1}\right)\right)+F_{1}\left(m\left(\mu_{q+1}, \mu_{q+2}\right)\right) \leq F_{1}\left(m\left(\mu_{q}, \mu_{q+1}\right)\right)
$$

and

$$
F_{2}\left(m\left(\mu_{q}, \mu_{q+1}\right)\right) \leq F_{1}\left(m\left(\mu_{q}, \mu_{q+1}\right)\right)+\xi\left(m\left(\mu_{q}, \mu_{q+1}\right)\right) .
$$

From Equation (50) and by monotonicity of $F_{1}$, we obtain that $\left\{m\left(\mu_{q}, \mu_{q+1}\right)\right\}$ is nondecreasing sequence. Hence, there exists $r \geq 0$ such that $m\left(\mu_{q}, \mu_{q+1}\right) \rightarrow r$ as $q \rightarrow \infty$. Assume that $r>0$; then, combining Equations (50) and (51) gives

$$
\begin{aligned}
F_{2}\left(m\left(\mu_{q+1}, \mu_{q+2}\right)\right) & \leq F_{1}\left(m\left(\mu_{q}, \mu_{q+1}\right)\right)+\xi\left(m\left(\mu_{q}, \mu_{q+1}\right)\right) \\
& -\pi\left(m\left(\mu_{q}, \mu_{q+1}\right)\right)
\end{aligned}
$$

From Equation (52), we get

$$
\begin{aligned}
F_{2}\left(L_{q+1}\right) & \leq F_{1}\left(L_{q}\right)+\xi\left(L_{q}\right)-\pi\left(L_{q}\right) \\
& \leq F_{1}\left(L_{q-1}\right)+\xi\left(L_{q}\right)+\xi\left(L_{q-1}\right)-\pi\left(L_{q}\right)-\pi\left(L_{q-1}\right) \\
& \vdots \\
& \leq F_{1}\left(L_{0}\right)+\xi\left(L_{q}\right)+\xi\left(L_{q-1}\right)+\cdots+\xi\left(L_{0}\right) \\
& -\pi\left(L_{q}\right)-\pi\left(L_{q-1}\right)-\cdots-\pi\left(L_{0}\right) .
\end{aligned}
$$

Let $\pi\left(L_{p_{n}}\right)=\min \left\{\pi\left(L_{0}\right), \pi\left(L_{1}\right), \cdots, \pi\left(L_{q}\right)\right\}$ and $\xi\left(L_{q_{n}}\right)=\max \left\{\xi\left(L_{0}\right), \xi\left(L_{1}\right), \cdots, \xi\left(L_{q}\right)\right\}$ for all $n \in$ $\mathbb{N}$. From Equation (53), we get

$$
F_{2}\left(L_{q+1}\right) \leq F_{1}\left(L_{0}\right)+q\left(\xi\left(L_{q_{n}}\right)-\pi\left(L_{p_{n}}\right)\right) .
$$

From Equation (48), we also get

$$
F_{2}\left(M\left(\mu_{q+1}, \mathcal{G} \mu_{q+1}\right)\right) \leq F_{1}\left(M\left(\mu_{0}, \mathcal{G} \mu_{0}\right)\right)+q\left(\xi\left(L_{q_{n}}\right)-\pi\left(L_{p_{n}}\right)\right) .
$$

Equations (12) and (55) $\operatorname{imply}_{\lim _{q \rightarrow \infty}} F_{2}\left(L_{q}\right)=-\infty$; thus, by (F2), $\lim _{q \rightarrow \infty} L_{q}=0$. Now, we prove that $\left\{\mu_{q}: \mu_{q} \in \mathcal{G} \mu_{q-1}\right\}$ is a Cauchy sequence. Since $F_{2}$ satisfies (F3), there exists $0<r<1$ such that

$$
\lim _{q \rightarrow \infty}\left(L_{q}\right)^{r} F_{2}\left(L_{q}\right)=0 .
$$

By Equation (54), we get for all $q \in \mathbb{N}$

$$
\begin{aligned}
\left(L_{q}\right)^{r} F_{2}\left(L_{q}\right)-\left(L_{q}\right)^{r} F_{1}\left(L_{0}\right) & \leq\left(L_{q}\right)^{r} q\left(\xi\left(L_{q_{n}}\right)-\pi\left(L_{p_{n}}\right)\right) \\
& \leq 0 .
\end{aligned}
$$

Letting $q \rightarrow \infty$ in Equation (57), we obtain

$$
\lim _{q \rightarrow \infty} n\left(L_{q}\right)^{r}=0
$$


This implies that there exists $q_{1} \in \mathbb{N}$ such that $q\left(L_{q}\right)^{r} \leq 1$, or, $L_{q} \leq \frac{1}{q^{1 / r}}$, for all $n>q_{1}$. Next, for $m>q \geq q_{1}$, we have

$$
m\left(\mu_{q}, \mu_{m}\right) \leq \sum_{i=q}^{m-1} m\left(x_{i}, x_{i+1}\right) \leq \sum_{i=q}^{m-1} \frac{1}{i^{1 / k}}
$$

since $0<k<1, \sum_{i=q}^{m-1} \frac{1}{i^{1 / k}}$ converges. Therefore, $m\left(\mu_{q}, \mu_{m}\right) \rightarrow 0$ as $m, q \rightarrow \infty$. Thus, $\left\{\mu_{q}: \mu_{q} \in \mathcal{G} \mu_{q-1}\right\}$ is a Cauchy sequence. Since $U$ is complete, there exists $\mu^{*} \in U$ such that $\mu_{q} \rightarrow \mu^{*}$ as $q \rightarrow \infty$. Since $F_{2}$ satisfies (F2), from Equation (55), we have

$$
\lim _{q \rightarrow \infty} M\left(\mu_{q}, \mathcal{G} \mu_{q}\right)=0
$$

From the hypothesis in Equation (2), we obtain

$$
0 \leq M(\mu, \mathcal{G} \mu) \leq \lim _{q \rightarrow \infty} \inf M\left(\mu_{q}, \mathcal{G} \mu_{q}\right)=0,
$$

which is a contradiction. Thus, $\mathcal{G}$ has a fixed point.

Theorem 6. Let $(U, m)$ be a complete metric space and $\mathcal{G}: U \rightarrow C(U)$ be a $F_{1}-F_{2}$-contraction satisfying the hypotheses in Equations (2) and (3) of Theorem 3. Assume that $F_{1}$ satisfies (F4), then $\mathcal{G}$ has a fixed point in $U$.

Proof. Assume that $\mathcal{G}$ has no fixed point in $U$. Let $\mu_{0} \in U$, then $M\left(\mu_{0}, \mathcal{G} \mu_{0}\right)>0$, otherwise $\mu_{0}$ is the fixed point of $\mathcal{G}$. Since $\mathcal{G}$ is $F_{1}-F_{2}$-contraction there exists $\mu_{1} \in T x_{0}$ with $M\left(\mu_{1}, \mathcal{G} \mu_{1}\right)>0$, otherwise $\mu_{1}$ is the fixed point of $\mathcal{G}$, satisfying

$$
\pi\left(m\left(\mu_{0}, \mu_{1}\right)\right)+F_{1}\left(M\left(\mu_{1}, \mathcal{G} \mu_{1}\right)\right) \leq F_{1}\left(M_{\mu_{0}, \mu_{1}}\right),
$$

and

$$
F_{2}\left(m\left(\mu_{0}, \mu_{1}\right)\right) \leq F_{1}\left(M\left(\mu_{0}, \mathcal{G} \mu_{0}\right)\right)+\xi\left(m\left(\mu_{0}, \mu_{1}\right)\right)
$$

where

$$
\begin{aligned}
M_{\mu_{0}, \mu_{1}}= & \max \left\{m\left(\mu_{0}, \mu_{1}\right), M\left(\mu_{0}, \mathcal{G} \mu_{0}\right), M\left(\mu_{1}, \mathcal{G} \mu_{1}\right),\right. \\
& \left.\frac{M\left(\mu_{1}, \mathcal{G} \mu_{0}\right)+M\left(\mu_{0}, \mathcal{G} \mu_{1}\right)}{2}\right\} \\
& \leq \max \left\{m\left(\mu_{0}, \mu_{1}\right), m\left(\mu_{0}, \mu_{1}\right), m\left(\mu_{1}, \mu_{2}\right),\right. \\
& \left.\frac{m\left(\mu_{1}, \mu_{1}\right)+m\left(\mu_{0}, \mu_{2}\right)}{2}\right\} .
\end{aligned}
$$

Since

$$
\begin{aligned}
\frac{m\left(\mu_{0}, \mu_{2}\right)}{2} & \leq \frac{m\left(\mu_{0}, \mu_{1}\right)+m\left(\mu_{1}, \mu_{2}\right)}{2} \\
& \leq \max \left\{m\left(\mu_{0}, \mu_{1}\right), m\left(\mu_{1}, \mu_{2}\right)\right\}
\end{aligned}
$$

it follows that

$$
M_{\mu_{0}, \mu_{1}} \leq \max \left\{m\left(\mu_{0}, \mu_{1}\right), m\left(\mu_{1}, \mu_{2}\right)\right\} .
$$

Since $F_{1}$ satisfies $(F 4)$, we obtain

$$
F_{1}\left(M\left(\mu_{1}, \mathcal{G} \mu_{1}\right)\right)=\inf _{\omega \in \mathcal{G} \mu} F_{1}\left(m\left(\mu_{1}, \omega\right)\right)
$$


Suppose that $m\left(\mu_{0}, \mu_{1}\right)<m\left(\mu_{1}, \mu_{2}\right)$; then, Equations (59) and (62) imply that

$$
\inf _{\omega \in \mathcal{G} \mu} F_{1}\left(m\left(\mu_{1}, \omega\right)\right) \leq F_{1}\left(m\left(\mu_{1}, \mu_{2}\right)\right)-\pi\left(m\left(\mu_{0}, \mu_{1}\right)\right) .
$$

Then, by Equation (63), there exists $\mu_{2} \in \mathcal{G} \mu_{1}$ such that

$$
F_{1}\left(m\left(\mu_{1}, \mu_{2}\right)\right) \leq F_{1}\left(m\left(\mu_{1}, \mu_{2}\right)\right)-\pi\left(m\left(\mu_{0}, \mu_{1}\right)\right),
$$

which is a contradiction. Hence, $M_{\mu_{0}, \mu_{1}} \leq m\left(\mu_{0}, \mu_{1}\right)$. Therefore, from Equations (59) and (62), we obtain

$$
F_{1}\left(m\left(\mu_{1}, \mu_{2}\right)\right) \leq F_{1}\left(m\left(\mu_{0}, \mu_{1}\right)\right)-\pi\left(m\left(\mu_{0}, \mu_{1}\right)\right)
$$

The rest of the proof follows as the proof of Theorem 5.

Theorem 7. Let $(U, m)$ be a complete metric space and $\mathcal{G}: U \rightarrow C(U)$. Assume that $F_{1}:(0, \infty) \rightarrow \mathbb{R}$ is a nondecreasing function, $F_{2}:(0, \infty) \rightarrow \mathbb{R}$ satisfies (F2) and (F3), and there exists $\pi:(0, \infty) \rightarrow(0, \infty)$ and $\xi:(0, \infty) \rightarrow(\xi, \infty), \xi>0$ such that, for all $\mu \in U$ with $M(\mu, \mathcal{G} \mu)>0$, we have $\omega \in \mathcal{G} \mu$ with $M(\omega, \mathcal{G} \omega)>0$ satisfying

$$
\pi(m(\mu, \omega))+F_{1}(M(\omega, \mathcal{G} \omega)) \leq F_{1}(M(\mu, \mathcal{G} \mu))
$$

and

$$
F_{2}(m(\mu, \omega)) \leq F_{1}(M(\mu, \mathcal{G} \mu))+\xi(m(\mu, \omega)) .
$$

If the hypotheses in Equations (2) and (3) of Theorem 3 hold, then $\mathcal{G}$ has a fixed point in $U$.

Proof. Assume that $\mathcal{G}$ has no fixed point in $U$. Let $\mu_{0} \in U$, we can construct a sequence $\left\{\mu_{q}\right\}$ in $U$ satisfying

$$
\pi\left(m\left(\mu_{q}, \mu_{q+1}\right)\right)+F_{1}\left(M\left(\mu_{q+1}, \mathcal{G} \mu_{q+1}\right)\right) \leq F_{1}\left(M\left(\mu_{q}, \mathcal{G} \mu_{q}\right)\right),
$$

and

$$
F_{2}\left(m\left(\mu_{q}, \mu_{q+1}\right)\right) \leq F_{1}\left(M\left(\mu_{q}, \mathcal{G} \mu_{q}\right)\right)+\xi\left(m\left(\mu_{q}, \mu_{q+1}\right)\right),
$$

$\mu_{q+1} \in \mathcal{G} \mu_{q}$ and $M\left(\mu_{q}, \mathcal{G} \mu_{q}\right)>0$. The rest of the proof follows as the proof of Theorem 5 .

\section{Notations and Setting of the Problem}

Consider the following nonlinear matrix equation

$$
V=\mathcal{Q}+\sum_{i=1}^{f} \mathscr{C}_{i} \varrho(V) \mathscr{C}_{i}^{*}-\sum_{j=1}^{k} \mathscr{D}_{j} \varrho(V) \mathscr{D}_{j}^{*},
$$

where $\mathcal{Q}$ is a positive definite matrix, $\mathscr{C}_{i}, \mathscr{D}_{j}$ are arbitrary $s \times s$ matrices for all $i=1, \ldots, f, j=1, \ldots, k$ and $\varrho$ is a self mapping on the set of all $s \times s$ Hermitian matrices, which maps set of all $s \times s$ Hermitian positive definite matrices into itself. Designate

$$
H(s)=\{V: V \text { is } s \times s \text { Hermitian matrix }\},
$$


which is a complete metric space in respect of the Ky Fan norm $\|.\|{ }_{1}$, defined by

$$
\|\mathscr{C}\|_{1}=\sum_{l=1}^{n} s_{l}(\mathscr{C})
$$

where $s_{l}(\mathscr{C}), l=1, \ldots, n$, are the singular values of $\mathscr{C}$. In addition,

$$
\|\mathscr{C}\|_{1}=\operatorname{tr}\left(\left(\mathscr{C}^{*} \mathscr{C}\right)^{1 / 2}\right),
$$

which is $\operatorname{tr}(\mathscr{C})$ for (Hermitian) nonnegative matrices and

$$
\mathcal{P}(s)=\{V \in H(s): V \text { is positive definite }\} .
$$

Define $\mathcal{G}: H(s) \rightarrow H(s)$ and $F: \mathbb{R}^{+} \rightarrow \mathbb{R}$ by

$$
\mathcal{G}(V)=\mathcal{Q}+\sum_{i=1}^{f} \mathscr{C}_{i} \varrho(V) \mathscr{C}_{i}^{*}-\sum_{j=1}^{k} \mathscr{D}_{j} \varrho(V) \mathscr{D}_{j}^{*}
$$

and $F(r)=\ln r$, respectively. Then, $F \in \mathfrak{F}$. For a function $\xi:(0, \infty) \rightarrow(\xi, \infty), \xi>0$ and $V \in U$ with $M(V, \mathcal{G} V)=\inf \{m(V, Y): Y \in \mathcal{G} V\}>0$, define the set

$$
\mathbb{F}_{\tilde{\xi}}^{V}=\left\{Y \in \mathcal{G} V: M(Y, \mathcal{G} Y)>0 \text { and } F(m(V, Y)) \leq F\left(M_{V, Y}\right)+\xi(m(V, Y))\right\},
$$

where

$$
M_{V, Y}=\max \left\{m(V, Y), M(V, \mathcal{G} V), M(Y, \mathcal{G} Y), \frac{M(Y, \mathcal{G} V)+M(V, \mathcal{G} Y)}{2}\right\}
$$

Note that a fixed point of $\mathcal{G}$ is a solution of Equation (70).

\section{Existence of Solution to Nonlinear Matrix Equations}

In this section, we prove the existence of the positive definite solution to the nonlinear matrix equation in Equation (70) by using the fixed point results in Section 2.

Theorem 8. Let $\varrho: H(s) \rightarrow H(s)$, which maps $\mathcal{P}(s)$ into $\mathcal{P}(s)$ and $\mathcal{Q} \in \mathcal{P}(s)$. Assume the following

(1) there exists a positive number $N$ for which $\sum_{i=1}^{f} \mathscr{C}_{i} \mathscr{C}_{i}^{*}+\sum_{j=1}^{k} \mathscr{D}_{j} \mathscr{D}_{j}^{*}<N I_{s}$; and

(2) for all $V, Y \in \mathcal{P}(s),\|\varrho(Y)-\varrho(V)\|_{1} \leq N^{-1}\left(M_{V, Y}\right) \exp \left(-\left(\frac{2\|Y-V\|+1}{2}\right)\right)$.

Then, Equation (70) has a solution in $\mathcal{P}(s)$.

Proof. Let $V \in H(s)$. For the functions $\pi:(0, \infty) \rightarrow(0, \infty)$ and $\xi:(0, \infty) \rightarrow(\xi, \infty)$ defined by $\pi(t)=t+\frac{1}{2}$ and $\xi(t)=\frac{1}{t}+\frac{1}{4}$, there exist $Y \in \mathbb{F}_{\tilde{\xi}}^{V}$ such that $Y=\mathcal{G} V$. Thus, for $M(V, \mathcal{G} V)>0$, Equation (11) holds true, $M(Y, T Y)>0$, and

$$
\begin{aligned}
F(m(V, Y)) & =F(m(V, \mathcal{G} V)) \\
& =\ln \left(\|\mathcal{G} V-V\|_{1}\right) \\
& <\ln \left(\|\mathcal{G} V-V\|_{1}\right)+\frac{1}{\|\mathcal{G} V-V\|_{1}}+\frac{1}{\|\mathcal{G} V-V\|_{1}} \\
& =F(M(V, \mathcal{G} V))+\xi(m(V, Y)) .
\end{aligned}
$$


Now,

$$
\begin{aligned}
M(Y, \mathcal{G} Y) & =m(\mathcal{G} V, \mathcal{G} Y) \\
& =\|\mathcal{G} Y-\mathcal{G} V\|_{1} \\
& =\operatorname{tr}(\mathcal{G} Y-\mathcal{G} V) \\
& =\operatorname{tr}\left(\sum_{i=1}^{f}\left(\mathscr{C}_{i} \varrho(V) \mathscr{C}_{i}^{*}-\mathscr{C}_{i} \varrho(Y) \mathscr{C}_{i}^{*}\right)+\sum_{j=1}^{k}\left(\mathscr{D}_{j} \varrho(V) \mathscr{D}_{j}^{*}-\mathscr{D}_{j} \varrho(Y) \mathscr{D}_{j}^{*}\right)\right) \\
& \leq \sum_{i=1}^{f}\left\|\mathscr{C}_{i} \mathscr{C}_{i}^{*}\right\|\|\varrho(V)-\varrho(Y)\|+\sum_{j=1}^{k}\left\|\mathscr{D}_{j} \mathscr{D}_{j}^{*}\right\|\|\varrho(Y)-\varrho(V)\| \\
& =\left[\sum_{i=1}^{f}\left\|\mathscr{C}_{i} \mathscr{C}_{i}^{*}\right\|+\sum_{j=1}^{k}\left\|\mathscr{D}_{j} \mathscr{D}_{j}^{*}\right\|\right]\|\varrho(Y)-\varrho(V)\| \\
& \leq \frac{\sum_{i=1}^{f}\left|\mathscr{C}_{i} \mathscr{C}_{i}^{*}\right|+\sum_{j=1}^{k}\left|\mathscr{D}_{j} \mathscr{D}_{j}^{*}\right|}{N}\left(M_{Y, V}\right) \exp \left(-\left(\frac{2\|Y-V\|+1}{2}\right)\right) \\
& <\left(M_{Y, V}\right) \exp \left(-\left(\frac{2\|Y-V\|+1}{2}\right)\right),
\end{aligned}
$$

and, thus,

$$
\begin{aligned}
\ln \left(\|\mathcal{G} Y-Y\|_{1}\right)=\ln \left(\|\mathcal{G} Y-\mathcal{G} V\|_{1}\right) & <\ln \left(\left(M_{Y, V}\right) e^{-\left(\frac{2\|Y-V\|+1}{2}\right)}\right) \\
& =\ln \left(M_{V, Y}\right)-\left\{\frac{2\|Y-V\|+1}{2}\right\}
\end{aligned}
$$

This implies that

$$
\|Y-V\|_{1}+\frac{1}{2}+\ln \left(\|\mathcal{G} Y-Y\|_{1}\right)<\ln \left(M_{V, Y}\right) .
$$

Consequently,

$$
\pi(m(V, Y))+F(m(Y, \mathcal{G} Y))<F\left(M_{V, Y}\right) .
$$

Thus, by using Theorem 3, we conclude that $\mathcal{G}$ has a fixed point and hence Equation (70) has a solution in $\mathcal{P}(s)$.

Corollary 8. Consider the matrix equation in Equation (70) with unitary matrices $\mathscr{C}_{i}, \mathscr{D}_{j}$ for all $i=1,2, \ldots, f$, $j=1,2, \ldots, k$. Assume that there exists a positive number $N$ for which $m+k<N$ and Hypothesis (2) of Theorem 8 holds for all $V, Y \in \mathcal{P}(s)$. Then, Equation (70) has a solution in $\mathcal{P}(s)$.

Corollary 9. Consider the matrix equation

$$
V=\mathcal{Q}+\mathscr{C} V \mathscr{C}^{*}-\mathscr{D} V \mathscr{D}^{*}
$$

Assume that there exists a positive number $N$ for which $\mathscr{C}_{i} \mathscr{C}_{i}^{*}+\mathscr{D}_{j} \mathscr{D}_{j}^{*}<N I_{s}$, and Hypothesis (2) of Theorem 8 holds for all $V, Y \in \mathcal{P}(s)$. Then, Equation (74) has a solution in $\mathcal{P}(s)$.

\section{Conclusions}

The motivation of the presented work is to get a new approach to the existence of the solution to nonlinear matrix equations via fixed point results for newly introduced multi-valued mappings, named as modified- $F$-contractions. It is also proved that our obtained results generalize and extend many 
existing results in the literature and nontrivial examples are provided to verify it. Here, we overcome the error mentioned in Example 1.1 of [9] by adopting a way other than that of Nguyen et al. In addition, we show that the main result of Nashine and Kadelburg [10] (see Theorem 2) is not a proper generalization of Feng and Liu's theorem by giving an example.

Author Contributions: All authors contributed equally and significantly in writing this paper. All authors have read and agreed to the published version of the manuscript.

Funding: This research received no external funding.

Conflicts of Interest: The authors declare that they have no competing interests.

\section{References}

1. Ran, A.C.M.; Reurings, M.C.B. A fixed point theorem in partially ordered sets and some applications to matrix equations. Proc. Am. Math. Soc. 2003, 132, 1435-1443. [CrossRef]

2. Duan, X.; Liao, A.; Tang, B. On the nonlinear matrix equation $X-\sum_{i=1}^{m} A_{i}^{*} X^{\delta_{i}} A_{i}=Q$. Linear Algebra Appl. 2008, 429, 110-121. [CrossRef]

3. Lim, Y. Solving the nonlinear matrix equation $X=Q+\sum_{i=1}^{m} M_{i} X^{\delta_{i}} M_{i}^{*}=Q$ via a contraction principle. Linear Algebra Appl. 2009, 430, 1380-1383. [CrossRef]

4. Berzig, M. Solving a class of matrix equations via the Bhaskar-Lakshmikantham coupled fixed point theorem. Appl. Math. Lett. 2012, 25, 1638-1643. [CrossRef]

5. Nadler, S.B. Multivalued contraction mappings. Pac. J. Math. 1969, 30, 475-488. [CrossRef]

6. Feng, Y.; Liu, S. Fixed point theorems for multi-valued contractive mappings and multi-valued Caristi type mappings. J. Math. Anal. Appl. 2006, 317, 103-112. [CrossRef]

7. Altun, I.; Minak, G.; Dağ, H. Multivalued F-contractions on complete metric space. J. Nonlinear Convex Anal. 2015, 16, 659-666.

8. Minak, G.; Olgun, M.; Altun, I. A new approach to fixed point theorems for multi-valued contractive maps. Carpathian J. Math. 2015, 31, 241-248.

9. Nguyen, V.L.; Phuong, L.T.; Hong, N.T.; Qin, X. Some fixed point theorems for multi-valued mappings concerning F-contractions. J. Fixed Point Theory Appl. 2018, 20, 139. [CrossRef]

10. Nashine, H.K.; Kadelburg, Z. Wardowski-Feng-Liu type fixed point theorems for multi-valued mappings. Fixed Point Theory, accepted.

11. Agarwal, R.P.; Hussain, N.; Taoudi, M.A. Fixed point theorems in ordered Banach spaces and applications to nonlinear integral equations. Abstr. Appl. Anal. 2012, 3, 15. [CrossRef]

12. Ahmad, J.; Hussain, N.; Khan, A.R.; Azam, A. Fixed point results for generalized multi-valued contractions. J. Nonlinear Sci. Appl. 2015, 8, 909-918. [CrossRef]

13. Asl, H.J.; Rezapour, S.; Shahzad, N. On fixed points of $\alpha-\psi$-contractive multifunctions. Fixed Point Theory Appl. 2012, 2012, 212. [CrossRef]

14. Ćirić, L.B. Fixed point for generalized multi-valued contractions. Mater. Vesn. 1972, 9, $265-272$.

15. Ćirić, L.B. Multi-valued nonlinear contraction mappings. Nonlinear Anal. Theory Methods Appl. 2009, 71, 2716-2723. [CrossRef]

16. Ding, H.S.; Kadelburg, Z.; Nashine, H.K. Common fixed point theorems for weakly increasing mappings on ordered orbitally complete metric spaces. Fixed Point Theory Appl. 2012, 2012, 85. [CrossRef]

17. Hussain, N.; Ahmad, J.; Azam, A. Generalized fixed point theorems for multi-valued $\alpha-\psi$-contractive mappings. J. Inequal. Appl. 2014, 2014, 348. [CrossRef]

18. Hussain, N.; Kutbi, M.A.; Salimi, P. Fixed point theory in $\alpha$-complete metric spaces with applications. Abstr. Appl. Anal. 2014. Available online: https://www.researchgate.net/publication/270671639_Fixed_ Point_Theory_in_-Complete_Metric_Spaces_with_Applications (accessed on 5 January 2020).

19. Hussain, N.; Mitrovic, Z.D. On multi-valued weak quasi-contractions in b-metric spaces. J. Nonlinear Sci. Appl. 2017, 10, 3815-3823. [CrossRef]

20. Imdad, M.; Gubran, R.; Arif, M.; Gopal, D. An observation on $\alpha$-type F-contractions and some ordered-theoretic fixed point results. Math. Sci. 2017, 11, 247-255. [CrossRef]

21. Iqbal, I.; Hussain, N. Fixed point theorems for generalized multi-valued nonlinear F-contractions. J. Nonlinear Sci. Appl. 2016, 9, 5870-5893. [CrossRef] 
22. Klim, D.; Wardowski, D. Fixed point theorems for set-valued contractions in complete metric spaces. J. Math. Anal. Appl. 2007, 334, 132-139. [CrossRef]

23. Mizoguchi, N.; Takahashi, W. Fixed point theorems for multi-valued mappings on complete metric spaces. J. Math. Anal. Appl. 1989, 141, 177-188. [CrossRef]

24. Nashine, H.K.; Kadelburg, Z.; Golubovic, Z. Common fixed point results using generalized altering distances on orbitally complete ordered metric spaces. J. Appl. Math. 2012, 2012, 12. [CrossRef]

25. Olgun, M.; Minak, G.; Altun, I. A new approach to Mizoguchi-Takahshi type fixed point theorems. J. Nonlinear Convex Anal. 2016, 17, 579-587.

26. Popescu, O. Some new fixed point theorems for $\alpha$-Geraghty contractive type maps in metric spaces. Fixed Point Theory Appl. 2014, 2014, 12. [CrossRef]

27. Salimi, P.; Latif, A.; Hussain, N. Modified $\alpha-\psi$-contractive mappings with applications. Fixed Point Theory Appl. 2013, 2013, 151. [CrossRef]

28. Samet, B.; Vetro, C.; Vetro, P. Fixed point theorem for $\alpha-\psi$-contractive type mappings. Nonlinear Anal. 2012, 75, 2154-2165. [CrossRef]

29. Türkoğlu, D.; Özer, O.; Fisher, B. Fixed point theorems for T-orbitally complete spaces. Mathematica 1999, 9, 211-218.

30. Wardowski, D. Fixed points of a new type of contractive mappings in complete metric spaces. Fixed Point Theory Appl. 2012, 2012, 94. [CrossRef]

31. Wardowski, D.; Dung, V.N. Fixed points of F-weak contractions on complete metric space. Demonstratio Math. 2014, 47, 146-155. [CrossRef]

32. Altun, I.; Minak, G.; Olgun, M. Fixed points of multi-valued nonlinear F-contractions on complete metric spaces. Nonlinear Anal. Model. Control 2016, 21, 201-210. [CrossRef]

33. Ćirić, L.B. Fixed point theorems for multi-valued contractions in complete metric spaces. J. Math. Anal. Appl. 2008, 348, 499-507. [CrossRef]

(C) 2020 by the authors. Licensee MDPI, Basel, Switzerland. This article is an open access article distributed under the terms and conditions of the Creative Commons Attribution (CC BY) license (http:/ / creativecommons.org/licenses/by/4.0/). 